\title{
Which is better for live birth prediction in patients aged over 40 with their first in vitro fertilization treatment?
}

\author{
Hee Jun Lee ${ }^{1}$, Yubin Lee ${ }^{1}$, Woo Sik Lee', Tae Ki Yoon ${ }^{1}$ \\ ${ }^{1}$ Fertility Center of CHA Gangnam Medical Center, College of Medicine, CHA University, Seoul, Korea
}

Problem statement: To evaluate clinical utility of antral follicle count (AFC) and anti-Müllerian hormone (AMH) in predicting in vitro fertilization (IVF) outcomes among the patients over 40 years old in their first IVF cycles.

Method: Total 219 patients aged 40 or older who underwent their first IVF with gonadotropin-releasing hormone antagonist protocol from January 2013 to September 2014 in CHA Gangnam fertility center were retrospectively analyzed. AFC and serum samples were measured prior to IVF treatment. The main outcomes were clinical pregnancy rate and live birth.

Results: 36 out of 219 patients achieved clinical pregnancy (16.4\%) and 27 out of 219 patients delivered (12.3\%). The receiver operating characteristic curve analysis to predict clinical pregnancy showed that both age and AFC equally had higher accuracy by area under the curve ( $A \cup C=0.657, P<0.01$ ) than serum $A M H$ (AUC $0.613, P=0.03)$. The optimum cut-off value of age was $\leq 41$ and that of AFC was $>3$ to predict clinical pregnancy. For the prediction of live birth, AFC had the highest accuracy (AUC 0.698, $P<0.01$ ), followed by age (AUC 0.674, $P<0.01$ ) and the number of total retrieved oocytes ( $A \cup C$ 0.620, $P=0.02$ ).
The optimum cut-off value of age was $\leq 41$, that of AFC was $>3$ and that of the number of total retrieved oocytes were $>6$. With multivariate regression analysis, age and $\mathrm{AMH}$ were significantly correlated with clinical pregnancy (age, odds ratio [OR] 0.53, $\mathrm{P}<0.01$; $\mathrm{AMH}$, OR 1.31, $\mathrm{P}=0.04)$, whereas age and $\mathrm{AFC}$ were association with live birth significantly (age, OR $0.41, P<0.01$; AFC, OR 1.10, $P=0.02$ ).

Conclusion: In patients aged over 40, AFC was shown to be a better biomarker than $\mathrm{AMH}$ for the prediction of live birth. $\mathrm{AMH}$ was positively correlated with clinical pregnancy but had no association with live birth. To predict the live birth, age $\leq 41$, AFC $>3$ and total retrieved oocytes $>6$ appeared to be meaningful. This study demonstrated the significance of AFC as a predictor of live birth for old aged women at their first IVF cycle with gonadotropin-releasing hormone antagonist protocol.

\begin{tabular}{|c|c|c|c|c|c|c|c|c|}
\hline & $\begin{array}{l}\text { Area } \\
\text { under } \\
\text { curve }\end{array}$ & $95 \% \mathrm{CI}$ & $\begin{array}{l}\text { Cut-off } \\
\text { value }\end{array}$ & Sensitivity & Specificity & $+\mathrm{LR}$ & -LR & P-value \\
\hline Age (yr) & 0.657 & $\begin{array}{l}0.59- \\
0.72\end{array}$ & $\leq 41$ & 93.9 & 38.7 & 1.53 & 0.16 & $<0.01$ \\
\hline $\mathrm{AFC}$ & 0.657 & $\begin{array}{l}0.59- \\
0.72\end{array}$ & $>3$ & 90.9 & 38.7 & 1.48 & 0.23 & $<0.01$ \\
\hline $\mathrm{AMH}(\mathrm{ng} / \mathrm{mL})$ & 0.613 & $\begin{array}{l}0.55- \\
0.68\end{array}$ & $>0.26$ & 97.0 & 24.2 & 1.28 & 0.13 & 0.03 \\
\hline $\begin{array}{l}\text { Basal FSH } \\
(\mathrm{mIU} / \mathrm{mL})\end{array}$ & 0.529 & $\begin{array}{l}0.46- \\
0.60\end{array}$ & $\leq 15.9$ & 93.6 & 21.1 & 1.20 & 0.31 & NS \\
\hline $\begin{array}{l}\text { No. of total } \\
\text { retrieved }\end{array}$ & 0.534 & $\begin{array}{l}0.47- \\
0.60\end{array}$ & $>6$ & 48.5 & 61.8 & 1.27 & 0.83 & NS \\
\hline
\end{tabular}

oocyte

Note: +LR: likelihood ratio positive. -LR: likelihood ratio negative. FSH: follicle stimulating hormone. AMH: anti-müllerian hormone. NS: not significant $(P>0.05)$. Values are given as mean \pm standard deviation

Table 2. Area under curve of ROC analysis for age, AFC, AMH, FSH, number of total retrieved oocyte and specific cut-off values for prediction of live birth

\begin{tabular}{|c|c|c|c|c|c|c|c|c|}
\hline 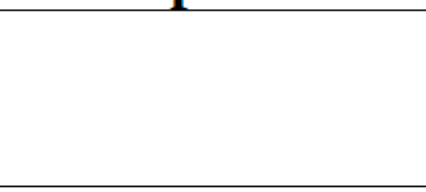 & $\begin{array}{l}\text { Area } \\
\text { under } \\
\text { curve }\end{array}$ & $95 \% \mathrm{CI}$ & $\begin{array}{l}\text { Cut-off } \\
\text { value }\end{array}$ & Sensitivity & Specificity & $+\mathrm{LR}$ & -LR & P-value \\
\hline Age (yr) & 0.674 & $0.61-0.74$ & $\leq 41$ & 96.0 & 37.3 & 1.54 & 0.11 & $<0.01$ \\
\hline $\mathrm{AFC}$ & 0.698 & $0.63-0.76$ & $>3$ & 92.0 & 37.6 & 1.48 & 0.21 & $<0.01$ \\
\hline $\begin{array}{l}\mathrm{AMH} \\
(\mathrm{ng} / \mathrm{mL})\end{array}$ & 0.609 & $0.53-0.66$ & $>0.26$ & 96.3 & 23.4 & 1.26 & 0.16 & NS \\
\hline $\begin{array}{l}\text { Basal FSH } \\
(\mathrm{mIU} / \mathrm{mL})\end{array}$ & 0.522 & $0.45-0.59$ & $\leq 15.9$ & 95.7 & 20.8 & 1.21 & 0.21 & NS \\
\hline $\begin{array}{l}\text { No. of total } \\
\text { retrieved } \\
\text { oocyte }\end{array}$ & 0.620 & $0.55-0.68$ & $>6$ & 59.3 & 63.0 & 1.60 & 0.65 & 0.02 \\
\hline \multicolumn{9}{|c|}{$\begin{array}{l}\text { Note: +LR: likelihood ratio positive. -LR: likelihood ratio negative. FSH: follicle stimulating hormone. AMH } \\
\text { anti-müllerian hormone. NS: not significant }(P>0.05) \text {. Values are given as mean } \pm \text { standard deviation }\end{array}$} \\
\hline \multicolumn{9}{|c|}{$\begin{array}{l}\text { Table } 3 \text {. Multivariate logistic regression analysis of the relationship between clinical pregnancy or live } \\
\text { birth and selected variables }\end{array}$} \\
\hline \multirow{2}{*}{\multicolumn{2}{|c|}{ Variables }} & \multicolumn{3}{|c|}{ Clinical pregnancy } & \multicolumn{4}{|c|}{ Live birth } \\
\hline & & \multicolumn{2}{|c|}{ Odds ratio $(95 \% \mathrm{CI})$} & $\mathrm{P}$ value & \multicolumn{3}{|c|}{ Odds ratio $(95 \% \mathrm{CI})$} & $\mathrm{P}$ value \\
\hline \multicolumn{2}{|l|}{ Age } & \multicolumn{2}{|c|}{$0.53(0.34-0.83)$} & $<0.01$ & \multicolumn{2}{|c|}{$0.41(0.23-0.73)$} & \multicolumn{2}{|c|}{$<0.01$} \\
\hline \multicolumn{2}{|l|}{$\mathrm{AFC}$} & \multicolumn{2}{|c|}{$1.07(0.98-1.17)$} & NS & \multicolumn{2}{|c|}{$1.10(1.02-1.19)$} & \multicolumn{2}{|c|}{0.02} \\
\hline \multicolumn{2}{|l|}{$\mathrm{AMH}$} & \multicolumn{2}{|c|}{$1.31(1.02-1.69)$} & 0.04 & \multicolumn{2}{|c|}{$1.22(0.91-1.65)$} & \multicolumn{2}{|c|}{ NS } \\
\hline \multicolumn{2}{|l|}{$\mathrm{FSH}$} & \multicolumn{2}{|c|}{$1.00(0.94-1.06)$} & NS & \multicolumn{2}{|c|}{$0.99(0.92-1.06)$} & \multicolumn{2}{|c|}{ NS } \\
\hline \multicolumn{2}{|c|}{ Total retrieved oocyte } & \multicolumn{2}{|c|}{$1.04(0.92-1.17)$} & NS & \multicolumn{2}{|c|}{$1.09(0.96-1.23)$} & \multicolumn{2}{|c|}{ NS } \\
\hline
\end{tabular}

